\title{
A Method for the Application of Numerical Simulations during Firefighting Operations Using Pre-Simulated, Model-Based Fire Scenarios
}

\author{
Puyan Abolghasem Zadeh", Uwe Rüppel \\ Institute for Numerical Methods and Informatics in Civil Engineering, Technische Universität Darmstadt, Darmstadt, Germany \\ Email: "puyan@puyanx.com
}

Received April 30, 2013; revised June 1, 2013; accepted June 9, 2013

Copyright (c) 2013 Puyan Abolghasem Zadeh, Uwe Rüppel. This is an open access article distributed under the Creative Commons Attribution License, which permits unrestricted use, distribution, and reproduction in any medium, provided the original work is properly cited.

\begin{abstract}
The spread of fire and smoke during a fire incident plays a crucial role in rescuing people from the burning building. So it is important for the decision makers (the head of rescue staff) to get a prediction about the spread of fire inside the building through computational techniques like numerical fire simulations. But these techniques require advanced mathematical knowledge and are very time consuming. This paper presents a new method which employs a set of presimulated and model-based scenarios to find the closest one to the real fire and present its results to the decision makers. For this purpose, we shift the performance consuming numerical fire simulations into a former phase by integration of these simulations into the planning process of the building. This is realized by enhancing the methods of Building Information Modeling (BIM). To provide the fire simulation results during a real case, our new concept includes a scenario database where all simulated fire scenarios will be collected. In a real case, a special search algorithm will go through this database to find the closest pre-simulated fire scenario to the real fire on the basis of reported information from the burning building.
\end{abstract}

Keywords: Fire Simulation; Model-Based Fire Scenario; Firefighting; Decision Making Support; Building Information Modeling

\section{Introduction}

Fire safety concepts are very significant for complex buildings with high number of occupants, like hospitals, airports, movie theaters, schools, shopping centers, etc. In case of fire in such buildings, it is very important for the rescue staff and the fire brigade to have a reliable prediction about the spread of fire inside the building, when they arrive at the scene of fire. This would help the responsible people in the decision making process and support them to take appropriate measures. These measures include rescue and evacuation as well as firefighting activities. The most dangerous threat to people in a building in case of a fire is mainly poisoning by smoke inhalation. Furthermore, the spreading of smoke inside buildings is faster than the spreading of fire. Brein and Hegger mention in [1] that $73 \%$ of fire victims were caused by smoke poisoning and only $5 \%$ by burning injuries. According to the ORBIT-Study [2], the probability of a

*Corresponding author. successful CPR (Cardiopulmonary Resuscitation) for a person with smoke poisoning is about $75 \%$ after 3 minutes. However, after 10 minutes this probability is only about $5 \%$. Therefore, it is very significant for the rescue staff on arrival at the operation scene to determine at what stage the fire is and to predict how the fire and smoke are developing in the next minutes inside the building.

A rescue and evacuation process begins generally with alerting the fire department as shown in Figure 1. Then some essential questions have to be answered there, like the fire location (address) or the number of required firefighters and fire engines.

After the crew arrives at the operation scene, the reconnaissance teams enter the burning building to collect information about the building occupants who are still inside as well as about the current state of the fire. This information will normally be reported via radio communication to the officer in charge (head of staff). On the basis of these reports and of the personal experiences, the 


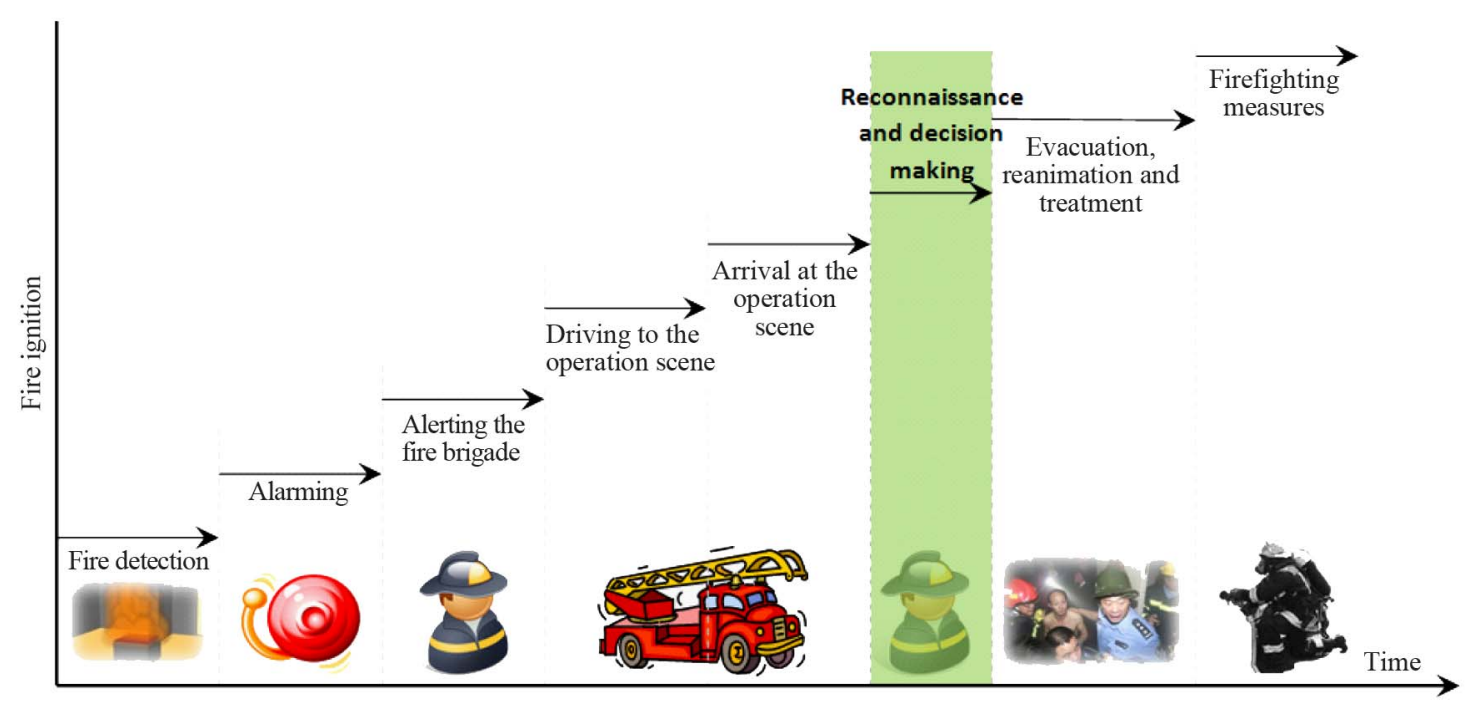

Figure 1. Processes in a firefighting operation.

officer in charge will develop a strategy to rescue the endangered people and carry out suitable firefighting measures.

The processes between alerting the fire department and arrival of firefighters at the operation scene are generally predictable and the crew can train them. In contrast to these processes, because each building has its own characteristics, the decision making process for suitable measures is highly variable and is very dependent on the personal experience of the head of staff with similar cases. The purpose of this paper is to describe a new method to support decision makers at this stage of rescue and firefighting operations in real time.

The new method uses a set of pre-simulated, modelbased fire scenarios to find the closest one to the real case and to employ its results to support the decision makers. For this purpose, a specific approach for providing the numerical simulation results should be developed and a novel search algorithm must be designed to find the closest pre-simulated scenario in real time. Figure 2 illustrates the details of our concept in an UML sequence diagram.

\section{Model-Based Numerical Fire Simulation}

With field models, complex physical and chemical relations in a fire can be described by using the fundamental equations of fluid dynamics. The process of solving these fundamental dynamics with numerical computer solutions is commonly referred as Computational Fluid Dynamics (CFD) [3]. CFD solvers divide the computational domain into a number of three-dimensional control volumes (finite elements or cells) and provide appropriate conservation equations (Navier-Stokes) for each control volume [4]. Deploying field models makes it possible to simulate precisely different aspects of a fire scenario, like spread of fire and smoke or local variation of different terms (e.g. temperature, pressure, visibility, etc.). Although field models have a high precision, they require advanced mathematical knowledge and high level of computing performance. This is why the numerical fire simulations with the current state of the art in science and technology cannot be deployed under firefighting operation conditions in real time [5].

With our new approach, we shift the simulation tasks to the planning phase of the building where enough time for required numerical simulations is available. One of the main requirements in our new approach is to provide as many pre-simulated fire scenarios as possible. This can significantly improve the quality of the determined pre-simulated scenario during the firefighting operation. Hence, a systematic manner for generating and executing of fire scenarios is needed which is integrated in the planning process of the building. With this manner, we can shift the performance consuming numerical fire simulation into a former phase of the building information management.

Nowadays a digital building design is a common approach in civil engineering. Particularly with the idea of BIM, it is possible to define fire scenarios based on the building models. It should be noted that there is not a consensus definition of BIM in technical literature. The Authors in [6] mention that both terms Building Information "Modeling" and Building Information "Model" can be referred to as BIM. They define these terms as follows:

- Building Information Modeling is the use of a computer model to simulate the construction and operation of a building. 


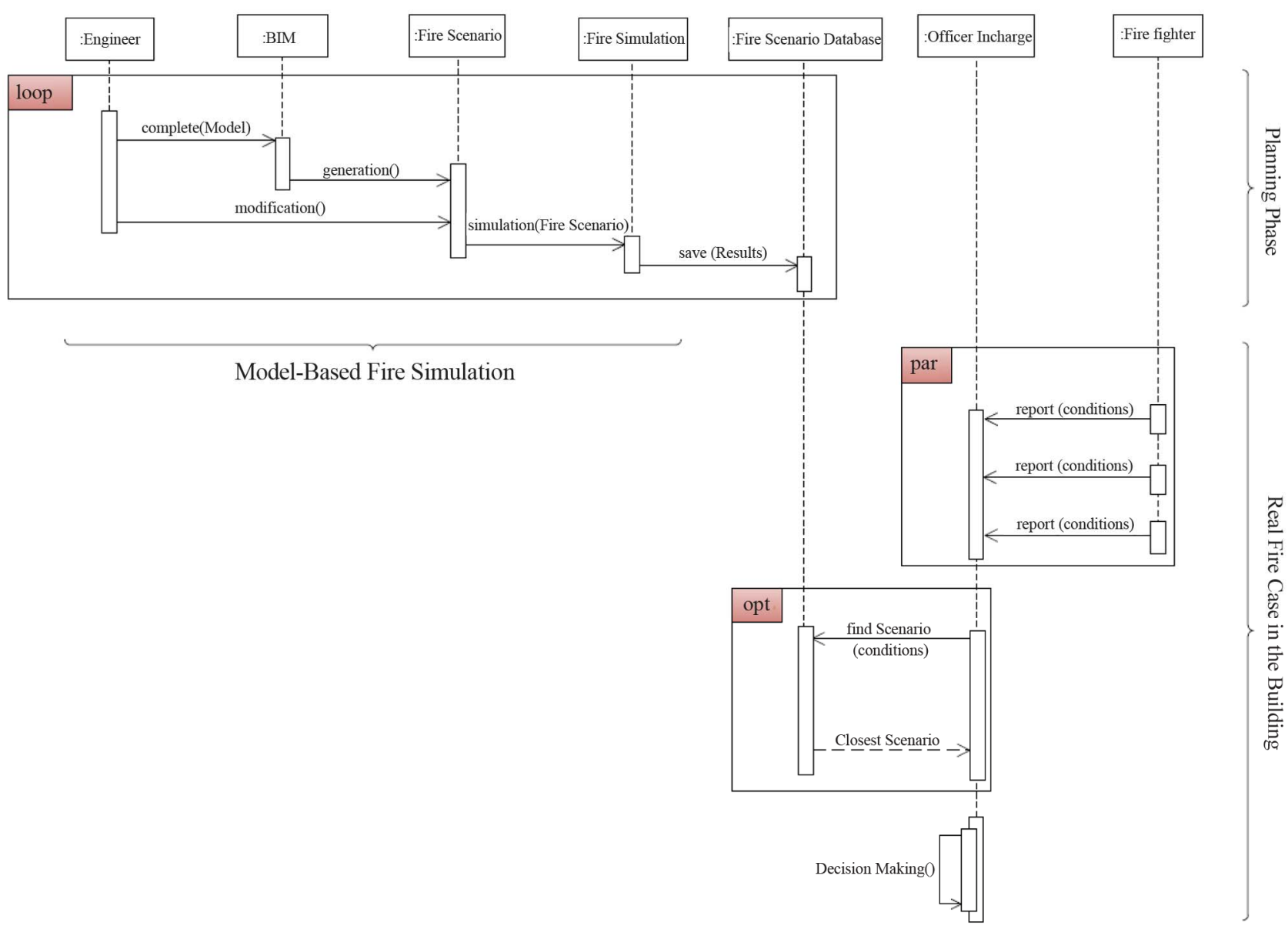

Figure 2. Concept for use of numerical simulations during firefighting operations using pre-simulated, model-based fire scenarios.

- Building Information Model is a data-rich, objectoriented, intelligent and parametric digital representation of the building. From such model can the required views and data be extracted and analyzed to generate information that can be used to make decisions and improve the process of delivering the building.

In [7], the authors describe BIM as a process for the generation, management and use of building information in an interoperable and reusable way throughout the building lifecycle, i.e., from planning and design through construction to the use of the building. Thus, BIM can be considered as a holistic model-based environment which collects all data and processes related to a building during its lifecycle. Furthermore, BIM can increase the collaboration between design disciplines and between designers and constructors [8].

In order to make a systematic generation of fire simulations possible, the advantages of BIM can be deployed. In former research work at the institute, we developed a new method for model-based fire safety analysis. This method is integrated in BIM as an additional application and allows to define and to simulate fire scenarios from the BIM environment [9]. With this application, fire scenarios can be configured based on the building model and carried out with an external numerical fire simulator. Thus, it is possible to systematically generate fire scenarios with this tool and simulate them in the planning phase where the factor time is not critical.

\section{Providing the Fire Simulation Results in a Scenario Database}

In order to support the decision making process during a firefighting operation, it is important to make predictions about the spread of fire and smoke for the next few minutes. Therefore, the simulation results for temperature and smoke density must at least be recorded for each scenario. Since measuring the smoke density in a live situation is difficult and there is a linear relation between smoke density and visibility [10], it will be reasonable to record for each fire scenario the simulation results for visibility instead of smoke density. For this purpose, we enhanced our application for model-based numerical fire simulations so that in each fire scenario a network of 
virtual sensors will be provided to record the local values for temperature and visibility (see Section 4).

As described above, a systematic model-based fire simulation during the planning of a building is realizable. After a simulation is finished, its results must be stored in a structured system in order to be used in a real firefighting operation. For this purpose, we developed a relational fire scenario database and enhanced our modelbased application so that the simulation results will be automatically transmitted into the scenario database after a simulation ends. The recorded data in the fire scenario database is as follows:

- General information about the fire scenario

- Room properties

- Fire source features

- Coordination of virtual sensor devices

- Simulated values for temperature and visibility at the location point of each virtual sensor (see Section 4).

\section{System Requirements and Design}

To record the local values for environmental parameters (temperature and visibility) at each simulation time step, a network of virtual sensors for each simulation area will be automatically generated through the model-based application. Hence, after a simulation ends, each sensor will contain the local development history of required environmental parameters (temperature and visibility). Later during a firefighting operation, the reported information from the burning building can be compared with these histories.

\subsection{Required Information from the Burning Building}

The following information must be reported through the communication (via radio or suitable sensors) between firefighters and the officer in charge:

- Position of fire source in the building

The reconnaissance teams should find out in which room is the fire source and in which rooms is fire already spread. This information will be used to determine the burning rooms in the building and compare them with pre-simulated scenarios in the database.

- Suspected burning material

The firefighters preferably should report the main burning material, like wood, chemicals, gases etc., or the main burning object, like furniture, carpet, cable, etc. As an alternative the usability of the burning room can be determined. So it is feasible to determine the equivalent Heat Release Rate Per Unit of Area (HRRPUA) through the burning material or the usability of the rooms. HRRPUA describes how big a fire source is [11]. Table $\mathbf{1}$ shows exemplary suggestions for HRRPUA in relation to the usability of the rooms.
Table 1. Suggestions for heat release rate per unit of area (HRRPUA) in relation to the usability of rooms according to [12].

\begin{tabular}{ccc}
\hline Room Usability & $\begin{array}{c}\text { HRRPUA } \\
\left(\mathbf{k W} / \mathbf{m}^{2}\right)\end{array}$ & Conditions \\
\hline Living Room & 250 & - \\
Office (Bureau) & 250 & - \\
School (Classroom) & 150 & $\begin{array}{c}\text { Wooden Furniture } \\
\text { and Seats } \\
\text { Wooden Seats, } \\
\text { Clothes and Bags }\end{array}$ \\
University (Lecture Room) & 130 & $\begin{array}{c}\text { - } \\
\text { Shopping Center }\end{array}$ \\
Library & 250 & With Metal Shelves \\
Wardrobe & 430 & approx. $12\left(\mathrm{~m}^{2}\right)$ \\
\hline
\end{tabular}

The information about the burning material can be used to correlate between strength of fire in the determined pre-simulated scenario and the recorded fires strength in the real case through the thermal sensors.

- Local visibility

The firefighters should report their visibility restriction through the radio. This information will be used in the search algorithm to find the current state of fire.

- Location point of firefighters and the local temperature

For these purposes, firefighters need to be equipped with suitable tracking and thermal sensors. We deploy the results of a former project at the institute for indoor navigation $[13,14]$ for tracking the location point of firefighters. Knowing the position of firefighters makes it possible to allocate the recorded local temperature and the reported local visibility. Similar to the local visibility, the local temperature will be used to determine the current stage of fire.

\subsection{Assignment of Recorded Parameters to a Location Point}

During real operations, at least one firefighter in each reconnaissance team should be equipped with a tracking system. With this system, the position of live recorded parameters from the burning building can be localized. In this concept, every captured firefighter will be surrounded by four virtual sensors that are available in all simulations. In the search process, a special algorithm first seeks these four surrounding virtual sensors and then finds the closest ones to the firefighter (tracking sensor) (Figure 3).

\subsection{Search Process}

Through a special search algorithm, the recorded simulation results for the nearest neighbor sensor in the scenario 


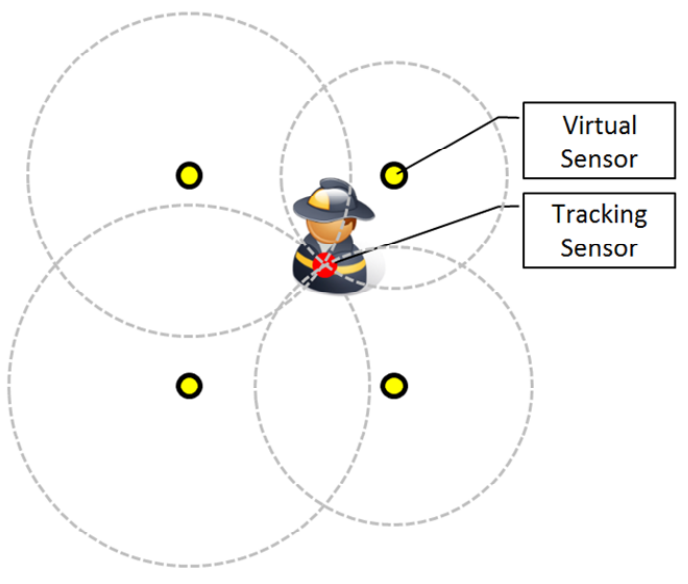

Figure 3. Assignment of a virtual sensor to a firefighter. database must be compared with the live records from the building. This procedure should be repeated for each captured firefighter inside the building. The pre-simulated scenario with the smallest deviation can be considered as a scenario with a similar fire and smoke development process to the real case in real time.

So its results can be delivered as the prediction of the environmental conditions. The activities for finding the closest scenario are shown in the UML diagram in Figure 4.

The search algorithm includes the following two basic steps:

1) Determination of the time frame for each presimulated scenario that matches with the current stage of fire in real case.

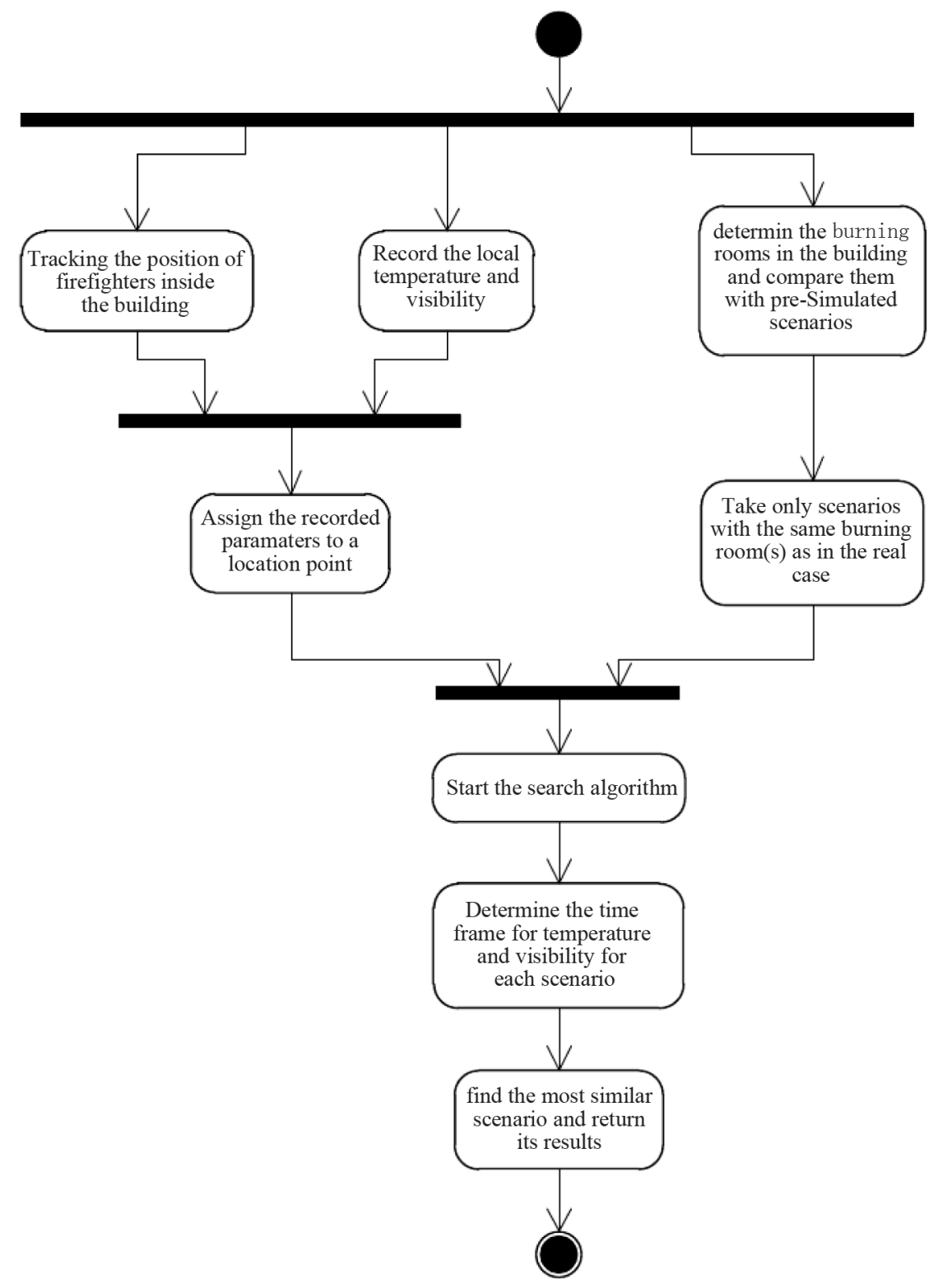

Figure 4. Search process for finding the closest pre-simulated numerical fire scenario to the real case in real time. 
2) Finding the most similar pre-simulated scenario to the real case based on the shortest calculated time frame.

Regarding the reported position of fire source and the burning material, a pre-selection on fire scenarios will be performed. It should be noted that because of the advanced development of the fire inside the building the position of fire source could not be clearly identified. In such case, all potential scenarios should be considered in the next step.

It is possible that none of the pre-simulated scenarios in the analysis meet the recorded local temperatures, despite matching the fire source position. That means that in the design phase, a fire scenario with a fire strength like in the real case is not considered. In cases like this, a correlation between temperature values (real and presimulated) is necessary.

In order to calculate the time frames for each scenario, the local pre-simulated values must be overlapped with the recorded ones. As mentioned before, we provide a virtual sensor network for each fire scenario and thus, each sensor contains a history of local variations of tem- perature and visibility in the associated scenario. Whereas the location of firefighters will be captured by the tracking system, it is feasible to find the closest virtual sensor to each firefighter to overlap its recorded and reported parameters with the sensors' histories in the database.

Through the overlapping of real and simulated values for each scenario, a time frame can be determined which represents the moment in which reports and records are carried out from the burning building. Hence, the narrower the time frame, the more the similarity between the fire scenario and the real case. Figure 5 shows an example of overlapping of recorded temperatures through three firefighters and the temperature histories from the scenario database for the closest virtual sensor to each of them.

It should be noted that the overlapping process can result multiple intersection points for each sensors history. In such cases, the search algorithm calculates all possible polygons and determines the narrowest one as the time frame of examined scenario. Figure 6 shows the deter-

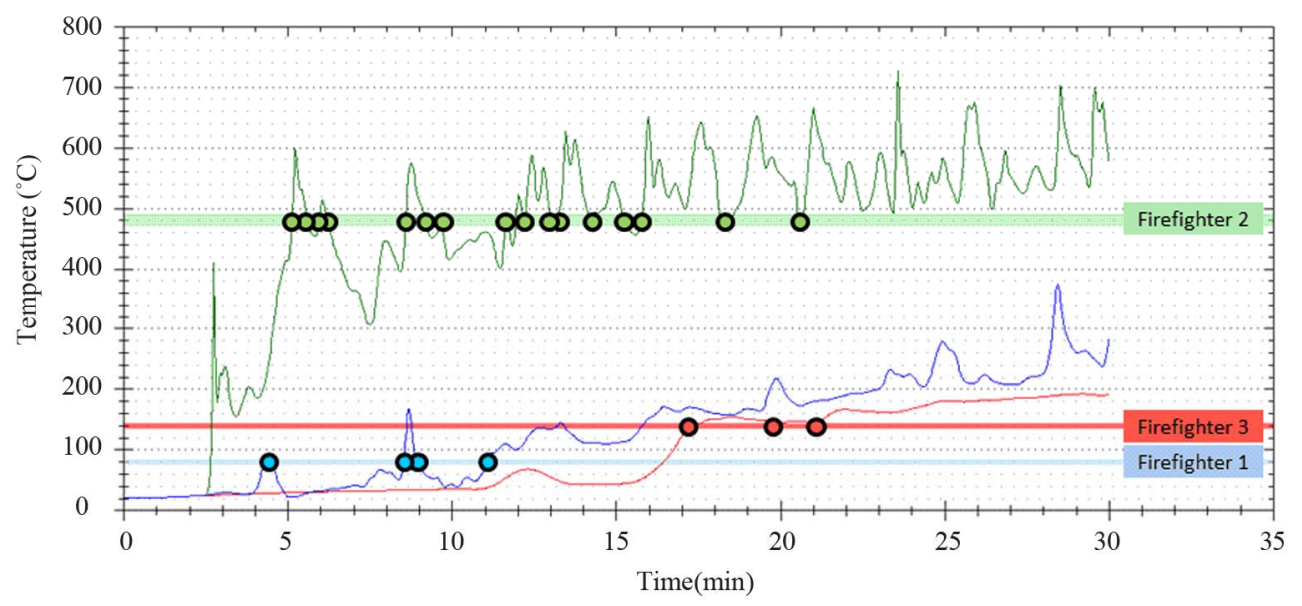

Figure 5. Intersections between recorded and simulated temperatures at the location point of three firefighters.

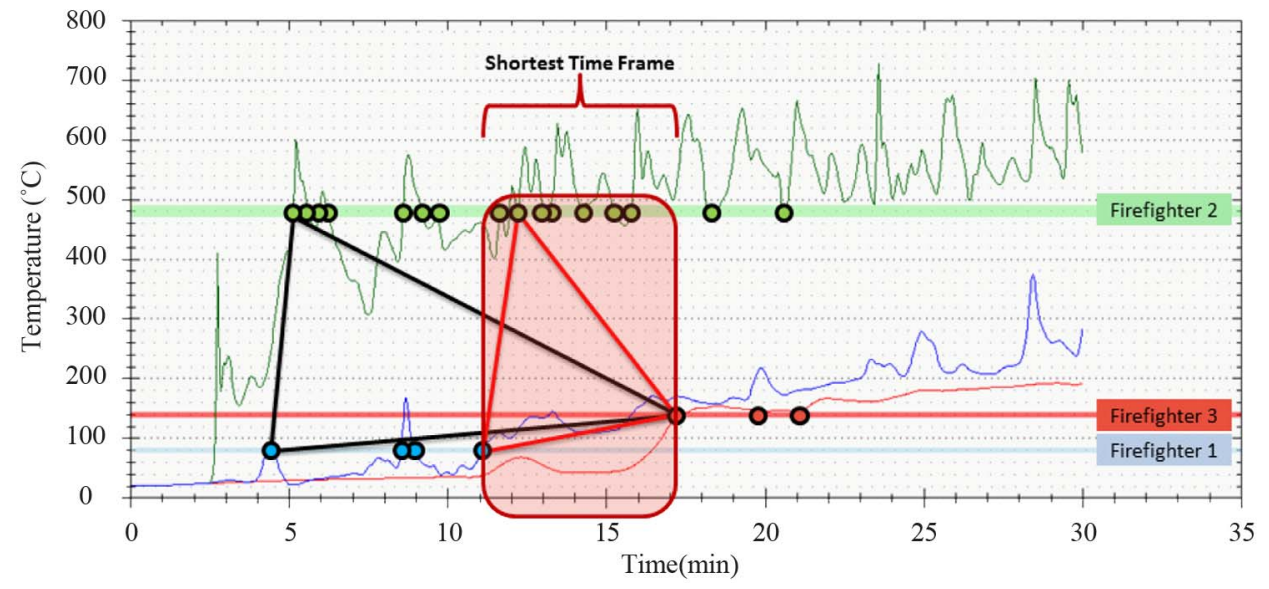

Figure 6. Shortest time frame for an inappropriate scenario. 
mined time frame for the previous example. The determined time frame here is about 6 minutes which disqualified the examined scenario.

The search algorithm will be carried out for each fire scenario for both parameters; temperature and visibility. After comparing the time frame with each other, the scenario with the shortest time window for both parameters will be chosen as the closest pre-simulated scenario to the real case. It is important that the determined time frames for temperature and visibility have to cover the same area on the time axis. This condition stands for the moment in which the records from the burning building are carried out. Figure 7 shows an example of the search process for a suitable scenario with a short time frame for temperature ( $<2 \mathrm{~min}$ ).

It is more likely that the search process has to deal with inaccurate reported information or faulty data records which both lead to certain deviations. The bigger the deviations, the wider the appropriate horizontal bar for real values of temperature and visibility and conesquently, the more intersection points and the longer required search time. It should be noted that the system works with only one record set from the burning building. In order to have more accurate results, the search process should be repeated for several record sets which is a time and performance intensive process. We do not suggest this step because it would be critical given the importance of time in emergency situations.

After the closest pre-simulated fire scenario is determined, its results can be requested from the scenario database and delivered to the officer in charge (the decision maker). According to the first tests with this system, the developed software application for this purpose is able to carry out the described processes within a few seconds [15]. Thus, with this application it is feasible to access the suitable results of a numerical simulation for the real fire case in real time.
It should be pointed out that there must be as many as possible pre-simulated scenarios in the planning phase provided and stored in the scenario database for more suitable results. The authors recommend that for each room there should be at least one fire scenario defined where the fire source is located in that room. Moreover, the simulation time for each scenario must be longer as usual to cover the operation time of the fire brigade. We suggest for this purpose a simulation time longer than 20 minutes.

\section{Hardware Design}

To use the new application in practice, a special portable computer device is needed. This device must be equipped with wireless Internet access as well as be light-weight and at the same time stable in order to be used during firefighting operations. For this purpose, we provide a tablet computer from "teXXmo KALEO" (Figure 8) with the following features [16]:

- Wireless LAN for data exchange between the database and the folder with simulation results

- A base weight of about $860 \mathrm{~g}$ which makes it easy to take anywhere

- Stable case with protective grip (rubber with a handstrap) that is suitable for outdoor applications

- A touch screen which makes it possible to be used also with firefighting gloves.

Other hardware properties are as following:

- CPU: Intel Atom Z530, 1.6 GHz

- RAM: 2 GB

- Storage: $2 \mathrm{~GB}$

Display Resolution: 800 x 600 (SVGA).

\section{Conclusions}

In emergency situations like fire, it is very significant for the rescue teams to have a reliable prediction about the

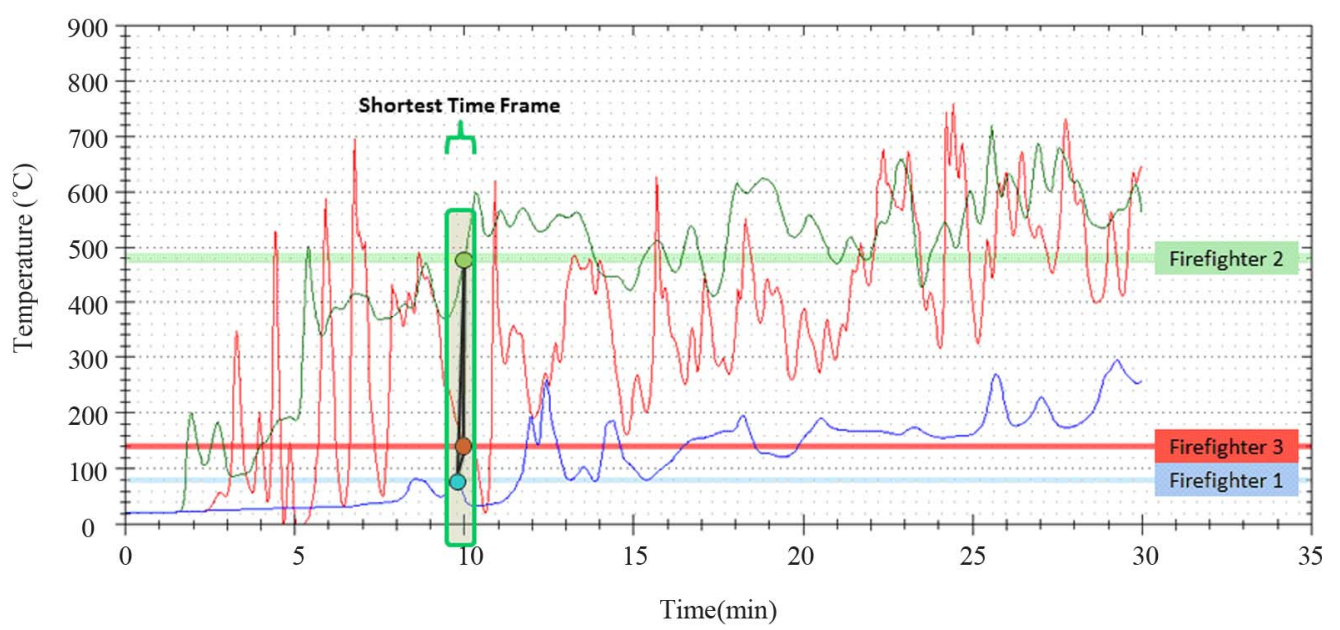

Figure 7. Determination of shortest time frame for an appropriate scenario. 


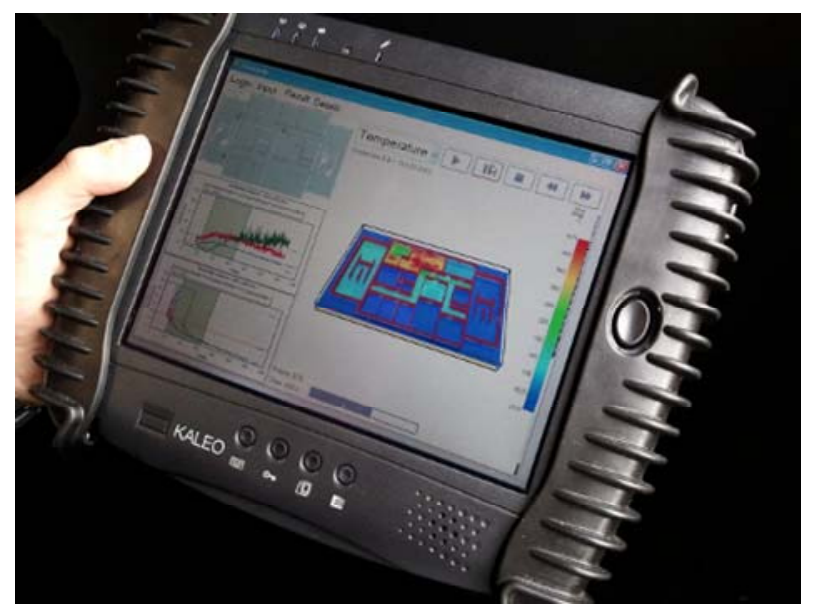

Figure 8. Tablet computer with the new application for using numerical fire simulations during firefighting operations.

spread of fire inside the building to make decisions about suitable firefighting measures. However, the decision making process is highly dependent on the personal experience of the head of staff with similar cases. This work presents a new method to support decision makers during firefighting operations in real time. This method makes it possible to employ results of numerical fire simulations during firefighting operations by finding the most similar pre-simulated fire scenarios to the real case based on received information from the burning building. It should be emphasized that the objective of this work is not to replace the role of decision makers or to have an exact prediction of real fire in a building. The purpose is to support the decision making process in critical emergency situations.

Although field models have a high precision and their use for our purpose will be useful, their simulation is time consuming and therefore, they cannot be deployed under firefighting operation conditions in real time. The new idea is to shift the required simulation tasks to the planning phase of the building where the factor time is not critical like in the emergency case. Thus, we enhanced the approaches in BIM in order to have systematic generation and execution of model-based fire scenarios. Furthermore, we developed a relational fire scenario database to store the simulation results in a structured manner and to use these results in a real firefighting operation.

The new method includes a special search algorithm to find the closest pre-simulated fire scenario from the database. The required input data for this algorithm should be collected through the reconnaissance teams from the burning building. With the search algorithm the similarity of each pre-simulated scenario to the real case can be determined. According to the first experiments with this system, the processing time of our new method is basi- cally short enough so that the use of numerical fire simulation results during firefighting operations (in real time) is possible.

\section{REFERENCES}

[1] D. Brein and T. F. Hegger, "Gefahrenpotentiale Summieren Sich,” Technical Report, 2002.

[2] P. Wibera, “ORBIT-Studie,” Technical Report, 1978.

[3] G. H. Yeoh and K. K. Yuen, "Computational Fluid Dynamics in Fire Engineering: Theory, Modelling and Practice,” Butterworth-Heinemann, Oxford, 2009.

[4] H. Czichos, M. Hennecke and Hütte "Das Ingenieurwissen,” 33rd Edition, Springer Verlag, Berlin, 2008.

[5] P. Abolghasemzadeh and U. Rüppel, "Pseudo Real-Time Fire Simulations with Building Information Models Using a Pre-Simulated Fire Scenario Database," Proceedings of the 2nd International Conference on Computational Engineering, Darmstadt, 2011, pp. 140-141.

[6] B. Ernstrom, D. Hanson, D. Hill, J. J. Clark, M. K. Holder, D. N. Turner, D. R. Sundt, L. S. I. Barton and T. W. Barton, “The Contractor's Guide To BIM,” The Associated General Contractors of America (AGC), 2006. http://www.agcnebuilders.com/documents/BIMGuide.pdf

[7] G. Lee, R. Sacks and C. M. Eastman, "Specifying Parametric Building Object Behavior (BOB) for a Building Information Modeling System,” Automation in Construction, Vol. 15, No. 6, 2006, pp. 758-776. doi:10.1016/j.autcon.2005.09.009

[8] G. V. Holness, "Building Information Modeling: Gaining Momentum,” ASHRAE Journal, Vol. 50, No. 6, 2008, pp. 28-41.

[9] U. Rüppel and P. Abolghasemzadeh, "BIM-Based Immersive Evacuation Simulations," Digital Proceedings of the 18. International Conference on the Application of Computer Science and Mathematics in Architecture and Civil Engineering (IKM 2009), Weimar, 2009.

[10] T. Jin, "Studies of Emotional Instability in Smoke from Fires,” Journal of Fire and Flammability, Vol. 12, 1981, pp. 131-142.

[11] V. Babrauskas, "Heat Release Rates,” In: SFPE Handbook of Fire Protection Engineering, 3rd Edition, National Fire Protection Association Quincy, 2002, pp. 1-3.

[12] D. Hosser, "Leitfaden Ingenieurmethoden des Brandschutzes,” Verein zur Förderung des Deutschen Brandschutzes eV (vfdb), 2009.

[13] U. Rüppel, P. Abolghasemzadeh and K. Stübbe, "BIMBased Immersive Indoor Graph Networks for Emergency Situations in Buildings," Nottingham University Press, Nottingham, 2010, pp. 65-71.

[14] K. Stuebbe, "Kontextsensitive Indoor-Navigation für Einsatzkräfte-Ortung, Wegberechnung, Zielführung und Einsatzkoordination,” Ph.D. Thesis, Shaker Verlag, Aachen, 2010.

[15] P. Abolghasemzadeh, "Ganzheitliche, Modellbasierte Ingenieurmethoden zu Hochperformanten Sicherheitsana- 
lysen in Gebäuden,” Ph.D. Thesis, Shaker Verlag, Aachen, 2012.

[16] teXXmo Mobile Solution GmbH \& Co. KG, "KALEO
Tablet PC Family,” 2012.

http://www.texxmo.de/index.php?cat=KALEO 